\title{
Immunoaffinity binding for separation of circulating tumor cells with microfluidic chips.
}

\author{
Hongmei Chen",2*, Baoshan $\mathrm{Cao}^{3}$, Hongda Chen², Xiaoqing $\mathrm{Lv}^{2}$, Lei $\mathrm{Li}^{2}$ \\ ${ }^{1}$ Division of Nanobionic Research, Suzhou Institute of Nano-Tech and Nano-Bionics, Chinese Academy of Sciences, \\ Suzhou, Jiangsu, 215123, China \\ ${ }^{2}$ Institute of Semiconductors, Chinese Academy of Sciences, Beijing, 100083, China \\ ${ }^{3}$ Department of Chemotherapy and Radiation Sickness, Peking University Third Hospital, 100191, China
}

\begin{abstract}
Immunoaffinity-based separation is high specific and could segregate different sizedcirculating tumor cells (CTCs), particularly for small sized-cells. Due to the big gap spacing between microposts, purity is relatively high, CTCs captured through collision and normal blood constitutes passing through, mitigating clogging. However, the complex structure and long modification procedure with expensive antibodies limited application of this approach. Immunoaffinity-based capture has the dilemma of EpCAM expressions in distinct carcinomas as the usual surface biomarker. For instance, in the common cell line used, EpCAM is highly expressed in MCF-7 (7287 antigens), HCT116 (6000 antigens), COLO205 (18560 antigens) and HT29 (13853 antigens), however, low expressed in HepG2 (2727 antigens), A549 (33 antigens) and Hela (8 antigens).
\end{abstract}

Keywords: Tumor cells, Microposts, Clogging, EpCAM, CTCs, COLO205, Hela.

Accepted on $23^{\text {rd }}$ May, 2017

\section{Introduction}

Immunoaffinity-based separation is high specific and could segregate different sized- circulating tumor cells (CTCs), particularly for small sized cells. Due to the big gap spacing between microposts, purity is relatively high, CTCs captured through collision and normal blood constitutes passing through, mitigating clogging. However, the complex structure and long modification procedure with expensive antibodies limited application of this approach. Immunoaffinity-based capture has the dilemma of EpCAM expressions in distinct carcinomas as the usual surface biomarker. For instance, in the common cell line used, EpCAM is highly expressed in MCF-7 (7287 antigens), HCT116 (6000 antigens), COLO205 (18560 antigens) and HT29 (13853 antigens), however, low expressed in HepG2 (2727 antigens), A549 (33 antigens) and Hela (8 antigens). This methodology has significant merit for the former, but it would lose effectiveness for low expression. Also, during metastasis, a subpopulation of tumor cells would undergo epithelial-to-mesenchymal transition (EMT), [1] a biological process having relevance in cancer progression. In EMT, tumor cells would down regulate their epithelial proteins such as E-cadherin and cytokeratins (CKs) and increase expression of mesenchymal proteins like $\alpha$-SMA and fibronectin [2]. This transition causes CTCs having spindleshape phenotype leading to invasion and metastasis. Thus CTCs experiencing EMT are relatively more malignant. Inevitably, those more invasive CTCs, going through EMT with severe malignancy of low epithelial expression, would be missed. Furthermore, flow rate or throughput really plays a significant role. Obviously, low flow rate, corresponding to prolonged collision, friction or interacting between CTCs and modified microstructures, would be conductive to capture. Conversely, increasing flow rate would reduce interaction time, thus decrease collision chances and affect capture efficiency. In order to facilitate capture and simplify complex microstructures of affinity-based microfluidic chips, size-based capture could be combined with antibody-modified microfluidic chips. This would ease the capture, release and enrichment.

Immunoaffinity binding is specific, extensively used approach in CTCs isolation based on Epithelial Cell Adhesion Molecular (EpCAM), a protein marker expressed on the surface of cells of epithelial origin in carcinoma cells [3-17]. The particular procedure is to modify inside surfaces of the microfluidic chip with specific antibody or aptamers [18-26]. When the blood sample flows through the microfluidic chip, antigen-antibody affinity bonding realizes capture. EpCAM is one of the most widely used surface biomarkers for positive selection of CTCs [27-29]. Negative capture aims at depleting leukocytes of immunomagnetic beads bonding thus attaining CTCs isolation. [1,29-32] Affinity binding includes antibody-antigen, Eselectin, aptamer $[33,34]$ and peptide binding.

\section{CTC-Chip}

Firstly, CTC-chip, [3] designed by Nagrath's group in 2007, consists of 78,000 high-aspect-ratio microposts functionalized with anti-EpCAM antibodies. The microstructure unit of CTCchip is composed of three microposts of $100 \mu \mathrm{m}$ diameters each arranged at three vertexes of an equilateral triangular, a 50 $\mu \mathrm{m}$ gap between microposts and a $50 \mu \mathrm{m}$ shift after every 3 rows. Modification is completed through treating the surfaces of CTC-chip with 3-mercaptopropyl trimethoxysilane, the coupling agent $\mathrm{N}-\gamma$-maleimidobutyryloxy succinnmide ester 
Citation: Chen H, Cao B, Li L. Immunoaffinity binding for separation of circulating tumor cells with microfluidic chips. J Mol Oncol Res.

2017;1(1):5-15.

(GMBS), NeutrAvidin and then biotinylated EpCAM. Expensive antibody could not avoid two days of modification. Modified microfluidic chip is not suitable to storage especially for a long time causing this method inconvenience except timely processing. Most of the following immunoaffinitybased isolation utilizing anti-EpCAM inherits this modification procedure. An average capture yield of over $65 \%$ could be achieved at 1-2 $\mathrm{ml}$ and 115 among 116 patient blood samples had been tested positive for CTCs. CTC-chip could achieve high capture yield, purity and throughput as the earliest immunoaffinity-based microfluidic chip in capturing CTCs utilizing EpCAM protein and EpCAM antibody bonding. In this affinity-based sorting, capture efficiency for this antibodycoated microfluidic chip is strongly dependent on the collision between CTCs and microstructures. However, for laminar flow at low Reynolds numbers, the linear streamlines deduce interaction chances of CTCs with antibody-coated surfaces especially in a large escaped area behind the microposts. As the earliest immunoaffinity binding for CTC isolation with microfluidic chip, this poses a high breakthrough to traditional separation approaches.

\section{HB-Chip}

To increase collision possibility, Scott group developed a herringbone structure (HB-Chip), a glass microscopic size, with eight microchannels patterned chevrons or herringbones on their upper surfaces [35]. This HB structure induces microvortices disrupting the laminar flow streamline that cells travel, utilizing the induced turbulent flow to increase the CTCs-affinity ligand interactions [36]. Capture efficiency is approximately $100 \%$ and CTCs were found in 14 of 15 patients with metastatic prostate and lung cancer. Comparing with CTC-chip, HB-Chip makes a major step forward in enhancing capture efficiency with this antibody-antigen binding method. Disrupting laminar flow producing microvortices is widely spread and the herringbone structure is further promoted and applied. Weian Sheng et al. developed a geometrically enhanced mixing chip (GEM chip) [37]. The staggered herringbone grooves interrupt streamlines and generate chaotic mixing and microvortex, maximizing collisions and interactions between target cells and device surfaces. CTCs were detected from 17 out of 18 samples from metastatic pancreatic cancer patients' blood with this GEM chip. Although collision and interaction has been enhanced greatly, increasing collision possibility to increase capture efficiency is more suitable for slow flow rate. Although HB-Chip enhanced capture efficiency dramatically, this chip could not prevent non-specific adhesion of white blood cells.

\section{Grapheme Oxide Chip}

This affinity-based functionization was further improved for sensitive capture of CTCs by Nagrath's group. [38,39] A grapheme oxide chip was developed with functionalized graphene oxide nanosheets on a flat substrate. 58,957 flowershaped gold patterns were designed on the silicon substrate and the distance between successive column structures is $150 \mu \mathrm{m}$. The graphene oxide nanosheets were subsequently modified by PL-PEG-NH2, Tetrabutylammonium (TBA) hydroxide, GMBS, NeutrAvidin and biotinylated EpCAM. Capture yield could reach $94.2 \%$ and $87.3 \%$ for $10-20$ and 100 spiked cells in $1 \mathrm{ml}$ whole blood, respectively. Functionized grapheme oxide nanosheets showed effective in isolating CTCs from blood samples of pancreatic, breast and lung cancer patients (Figure $1 \mathrm{~A}-\mathrm{G})$. In this graphene oxide chip, traditional modification process has been altered. 

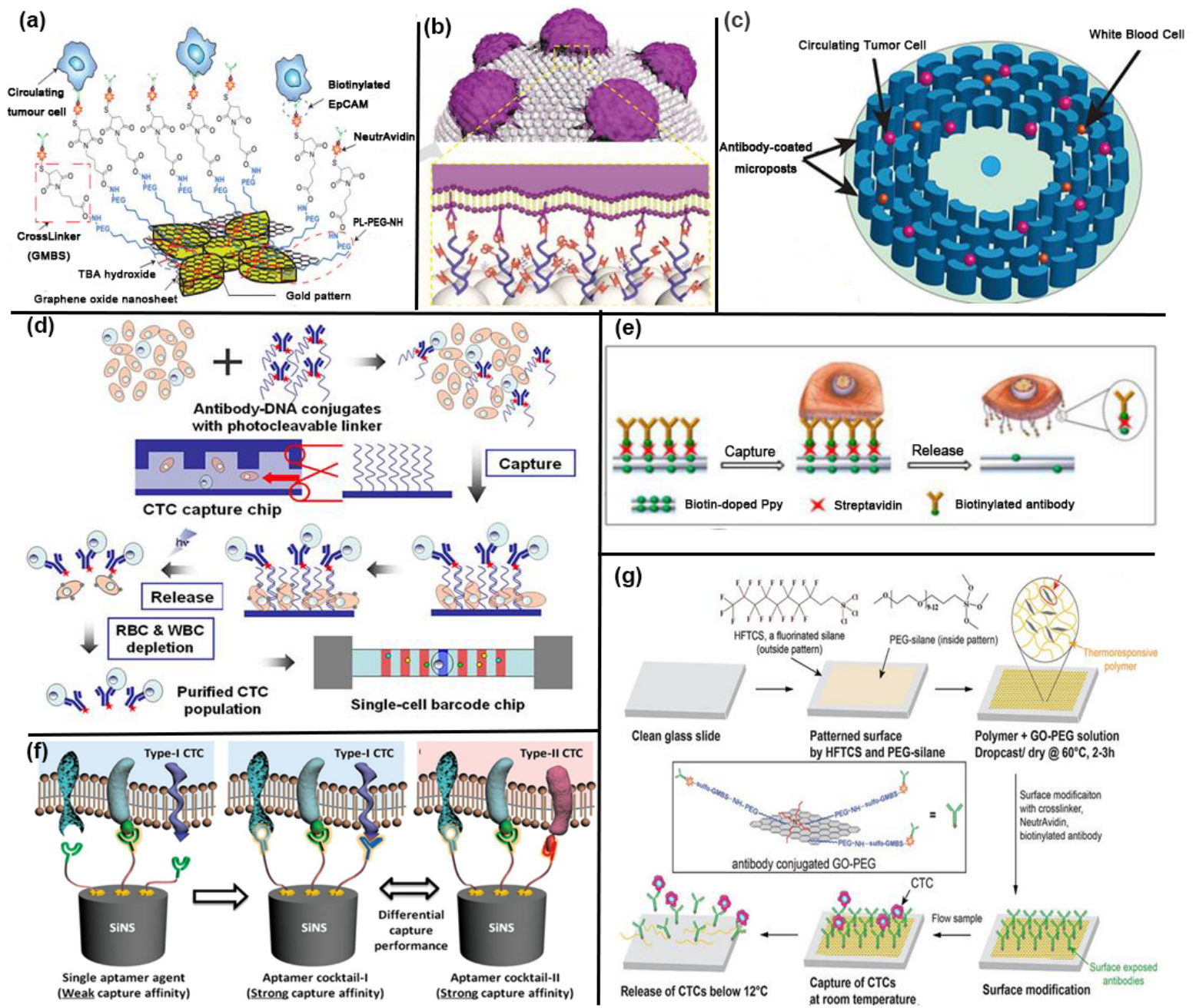

Figure 1: Representative microfluidic chips using antigen-antibody affinity to capture CTCs (A) Functionalized grapheme oxide nanosheets (B) Apatamer functionalized nanostructure surface of barcode particles (C) Onco Bean Chip (D) Photocleavable ssDNA-encoded antibody conjugated single-cell barcode chip (E) Conducting Polymer-deposited microfluidic device (F) A silicon nanowiree substrate (SiNS) embedded microfluidic chip $(\mathrm{G})$ Tunable thermal-sensitive polymer-graphene oxide (GO) microfludic device.

\section{Onco Bean Chip}

Murlidhar and coworkers in Nagrath's group demonstrated a radial flow microfluidic device, Onco Bean Chip [40]. Onco Bean Chip provides varying shear across the device, for affinity-based isolation of CTCs at $10 \mathrm{ml} / \mathrm{h}$. Bean-shaped microposts are arranged in a circular array and subsequent arrays are rotated and shifted. Linear laminar flow has been replaced, varying shear rates produced at each radius. Since modification effect varies in radial direction. Cells with different surface antigen expressions would be captured at an optimal shear rate location. Flow rate reduces in the radial direction, CTCs expressed high antigen would be trapped in the region close to the center, while more concentrated in the peripheral region with low antigen expression, where prolonged interaction increases collision. Mean efficiency is over $80 \%$ and viability is $93 \%$ at this high flow rate. This rigorous Onco Bean Chip was validated for 6 clinical patient samples comparing with CTC-chip. This is a rapid progress in the flow rate where the antibody-based method is restricted. Anti-EpCAM modification could be combined with fabricating a structural surface resembling morphology of CTCs to increase capture efficiency (Figure 1C). This is an exquisite break of limited flow rate frequently met in this immunoaffinity-based approach and enables more efficient processing of higher volumes of blood.

\section{FSMW}

In 2012, Nadia Saucedo-zeni and coworkers from Germany reported a structured medical Seldinger guidewire (FSMW) for in vivo isolation of CTCs from peripheral blood of cancer patients [41]. FSMW was deposited with a $2 \mu \mathrm{m}$ thick gold layer, subsequently attached with a hydrogel layer composed of a linear, synthetic polycarboxylate, and then functionalized with EDC (1-ethyl-3-[3-dimethylaminopropyl] carbodiimide hydrochloride) and NHS (N-hydroxysuccinimide) via covalent coupling of a chimeric antibody, which could bind with the epithelial cell adhesion molecule CD326, antigen of most CTCs. FSMW was placed into the cubital vein of a cancer patient for $30 \mathrm{~min}$ with the EpCAM-antibody-functionalized FSMW surface in length of $2 \mathrm{~cm}$ immersed in the blood flow within the lumen of the vein. Specificity and sensitivity of FSMW had been tested in 24 breast cancer or non-small cell 
Citation: Chen H, Cao B, Li L. Immunoaffinity binding for separation of circulating tumor cells with microfluidic chips. J Mol Oncol Res. 2017;1(1):5-15.

lung cancer (NSCLC) patients and in 29 healthy volunteers. 22 of 24 patients had been identified with EpCAM-positive CTCs of a median of $5.5(0-50)$ CTCs in breast $(n=12)$ and 16 (2-515) CTCs in NSCLC $(n=12)$ across all tumor stages including early stage cancer. The GILUPI CellCollector is the application of this FSMW. It has two generation of DC01 with $2 \mathrm{~cm}$ long functionalized tip in $2 \mathrm{~cm}$ length and DC02 consisting of three tin twisted stainless steel wires. The in vivo applied GILUPI CellCollector DC01 shows a CTC detection frequency of $70 \%$ in non-metastatic patients and $72 \%$ in metastatic patients comparing to $12 \%$ and $49 \%$, respectively, from in vitro system CELLSEARCH. It has applications in CTCs enumeration, immunofluorescence analysis of proteins of interest (e.g. HER2, ALK, AR-V7), gene mutation analysis (e.g. EGFR, KRAS), fluorescence in situ hybridization (FISH) and next generation sequencing (NGS). Comparing with other microfluidic chips, definitely FSMW has the highest possibility to capture CTCs in vivo, but this in vivo test would possibly produce certain uncomfortable to patients.

\section{Thermal GO-Polymer Device}

As advanced progress to conventional materials used, lately, Hyeun Joong Yoon and coworkers in the group of Sunitha Nagrath proposed a thermal-controllable sensitive polymergraphene oxide composite film (Polymer-GO composite film) deposited on the glass substrate combined with a PDMS chamber to form a GO-polymer device [42]. The same modification procedure above was followed with anti-EpCAM immobilized on the surface. When the temperature is over a lower critical solution temperature (LCST) of approximately 13 , the film is insoluble, inducible to cell capture. Inversely, below LCST, the film is soluble, which facilitates release. For MCF-7 cells spiked into the buffer, the highest capture rate could reach $95.21 \%$ at a flow rate of $1 \mathrm{mLh}^{-1}, 95.21 \%$ and $91.56 \%$ of cells could be released in buffer and blood experiments, respectively, and $91.68 \%$ of cells could be kept viable (Figure 1E). Innovatively, through controlling variation of temperature this thermal GO-polymer device facilely realizes capture and release.

\section{Cell-SELEX Derived Aptamers with a Silicon Nanowire Substrate (SiNS) Embedded Microfluidic Chip}

Assays have been performed for NSCLC patients using different combination of cell-SELEX derived aptamers with a silicon nanowire substrate (SiNS) embedded microfluidic chip by Libo Zhao and colleague [43-46]. Capture performances are over $50 \%$ for one single aptamer and three cocktails, Ap1, Cocktail A (Ap1+Ap2), Cocktail B (Ap1+Ap2+Ap4) and Cocktail C (Ap1+Ap3+Ap4). Unbelievably, this group has been tried on 11 stage-IV NSCLC patients and treatment monitoring (Figure 1F).

\section{Barcode Particles Assembled by Colorful Silica Nanoparticles}

In 2014, Fuyin Zheng and coworkers fabricated barcode particles assembled by colorful silica nanoparticles. [47] This could offer large surface area allowing immobilized and reacted modification and a hemispheric array surface similar to topography of cells. Decoration is completed with dendrimer and DNA aptamers (TD05, Sgc8, and Sgd5). Capture specificities for the Ramos and CCRF-CEM cells turn out to be $98 \%$ and $97 \%$, respectively. $86.6 \%$ captured cells could be released (Figure 1B).

\section{A Conducting Polymer-Deposited Microfluidic Device}

In 2014, Seung Hyun Jeon and coworkers designed a conducting polymer-deposited microfluidic device enabling CTCs recovery with high purity [48]. The modification was completed with anti-EpCAM antibody with a technology of electrochemical deposition of biotin-doped polypyrrole (Ppy), subsequently added the assembly of streptavidin and biotinylated antibody. CTCs could be recognized by topographical features of the channel surface. Furthermore, the designed negative electric voltages could release captured CTCs into floating in the fluid and positive voltages would cause electric field-induced damage to the cytoskeleton and nuclear membrane as WBCs. The anti-EpCAM-coated biotin/ Ppy-microfluidics was utilized to capture EpCAM-positive HCT116 and EpCAM-negative T24. Over 90\% capture rate could be reached with $50 \mu \mathrm{g} / \mathrm{mL}$ antibody used regardless throughput. At a flow rate of $1.2 \mathrm{~mL} / \mathrm{h}, 80 \%$ of the captured cells were accumulated in the first $8 \mathrm{~cm}$ of the channel. For artificial blood sample of MCF7 cells spiked into $1 \mathrm{~mL}$ whole blood, mean CTC capture and release rate were $97 \%$ and $95 \%$ at $1.2 \mathrm{~mL} / \mathrm{h}$, respectively. Performance of this biotin-doped Ppy-deposited microfluidic system was dramatically enhanced with a mixture of biotinylated antibodies of anti-EpCAM, antiTROP2, anti-EGFR, anti-N-Cadherin, and anti-vimentin (Figure 1G). Thorough exploring of electrochemical deposition and mixture of different antibodies modification produce solid results for a certain antibody concentration.

\section{An Integrated Microfluidic Chip System}

Recently, Yuliang Deng and coworkers reported an integrated microfluidic chip system [49]. Photocleavable ssDNA-encoded antibody conjugates and HB microfluidic chip are generated to capture CTCs. Slight UV irradiation release discriminated CTCs. After RBCs and WBCs are negatively depleted, a single-cell barcode chip (SCBC) integrated with an enhanced poly-L-lysine (PLL) barcode pattern to isolate high purityCTCs for profiling a panel of functional proteins. Close to $93 \%$ capture efficiency could be reached for HCT116 cells spiked and $86 \%$ in whole blood at $1 \mathrm{ml} / \mathrm{h}$ with release efficiency of $90 \%$ and viability of $88 \%$ (Figure 1D). Two steps combined to reach high capture efficiency, high purity and high viability. Various immunoaffinity-based separating microfluidic chips have been listed in Table 1. 
In summary, immunoaffinity binding is specific depending on bond of antigen and antibody such as EpCAM and antiEpCAM. This has significance in capturing or isolating circulating tumor cells with microfluidic chips composed of surface modified microposts. Beyond this wonderful specific "liquid biopsy", there should be much other significance. Modified with some specific antibody, aptamer, polysaccharide or polypeptide on some non-poisonous nanoparticles such as ferri ion, exosome or ctDNA has high possibilities in binding with those modified nanoparticles emitting photoluminescence. After tracing trajectory of those nanoparticles, they should appear as a tail. Following tracing source of those nanoparticles with fluorescence, we should be able to find specific site of tumor happening. Otherwise, from absorption spectroscope of those nanoparticles, analyzing or decomposing with perhaps Inductively Coupled Plasma Mass Spectrometry (ICP-MS) or Angele resolved photoemission spectroscopy (ARPES) should offer us much biological molecular information about protein composition of exosome, ctDNA and
CTCs. This would be critical in early diagnosis and therapy. In fact, many researchers regard CTCs for metastasis. They are greatly effective for this stage. However, CTCs with extremely rare number of CTCs detected belong to early stage before generation of second tumor, which significantly enhanced CTCs research status. More importantly, we could substitute traditional immunofluorescence with optics, install software into portable electronic decoration and illuminate the patient blood samples with light. From the spectroscopy shown the screen of those electronic decorations, we should be able to know whether we had cancer, the type of cancer and specific information of cancer and treatment plan would come out from the doctor. Usually we put high emphasis on patient samples. If no CTCs, exosome or ctDNA detected, that would be everybody needed. Normal checking for normal persons before reminding doctors to take measurements to treat would be routine inspection and a big prevention and market of CTCs product invests. Immunoaffinity binding for CTCs isolation play a key in prognosis and diagnosis of cancer.

Table 1: Summary of affinity-based microfluidic chip technology for CTCs isolation.

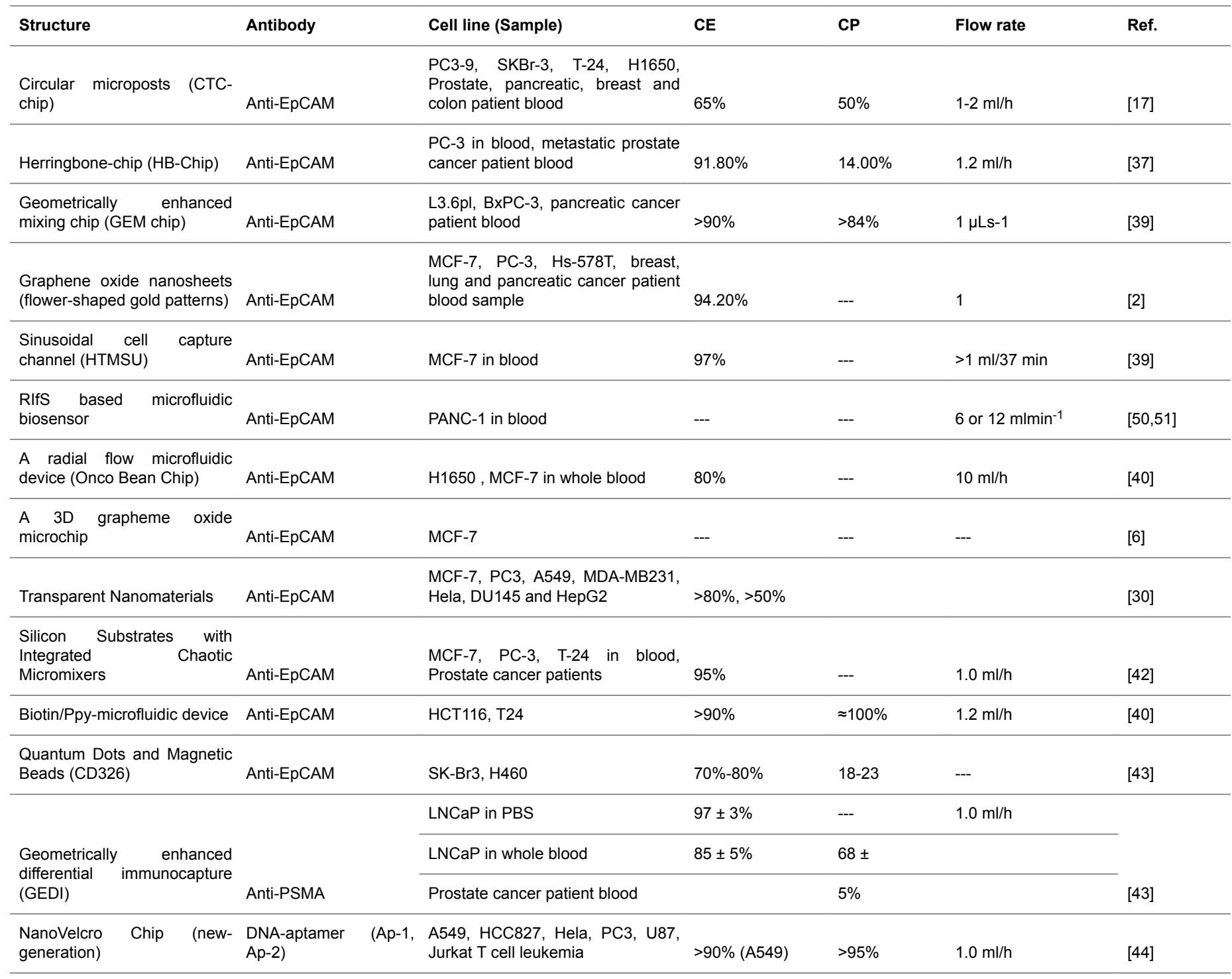


Citation: Chen H, Cao B, Li L. Immunoaffinity binding for separation of circulating tumor cells with microfluidic chips. J Mol Oncol Res. 2017;1(1):5-15.

\begin{tabular}{|c|c|c|c|c|c|c|}
\hline Aptamer microchip & DNA-aptamer & $\begin{array}{l}\text { In RPMI medium: CCRF-CEM, } \\
\text { NB-4 }\end{array}$ & $>80 \%$ & $\approx 97 \%$ & $0.72 \mathrm{ml} / \mathrm{h}$ & [52] \\
\hline \multirow{3}{*}{$\begin{array}{l}\text { A serpentine microfluidic chip } \\
\text { with S-channel separated into } \\
\text { three regions }\end{array}$} & \multirow{3}{*}{$\begin{array}{l}\text { DNA-aptamer } \quad(S g c 8, \\
\text { TD05, Sgd5) }\end{array}$} & CEM & $83 \pm 9 \%$ & $97 \%$ & \multirow[b]{3}{*}{$1.0 \mathrm{ml} / \mathrm{h}$} & \multirow[b]{3}{*}{ [41] } \\
\hline & & Ramos & $61 \pm 14 \%$ & $97 \%$ & & \\
\hline & & Toledo cells & $50 \pm 10 \%$ & $88 \%$ & & \\
\hline SELEX Aptamer probe & Aptamer BC-15 & $\begin{array}{l}\text { PL-45, MCF-7, A549, MDA- } \\
\text { MB-231, HT-29, MCF10A }\end{array}$ & --- & --- & --- & [42] \\
\hline $\begin{array}{l}\text { an integrated microfluidic chip } \\
\text { system }\end{array}$ & ssDNA & HCT116 & $93 \%$ & --- & 88 & {$[52]$} \\
\hline Barcode Particles & TD05, Sgc8, Sgd5 & Ramos, CCRF-CEM & $98 \%-97 \%$ & --- & --- & [43] \\
\hline $\begin{array}{l}\text { A SiNS embedded } \\
\text { microfluidic chip }\end{array}$ & $\begin{array}{l}\text { cell-SELEX } \\
\text { aptamers }\end{array}$ & $\begin{array}{l}\text { A549, H460, H292, H1299, SK- } \\
\text { MES-1 }\end{array}$ & $80 \%$ & --- & --- & [20] \\
\hline
\end{tabular}

\section{References}

1. Dave B, Mittal V, Chang J, et al. Epithelial-mesenchymal transition, cancer stem cells and treatment resistance. Breast Cancer Research. (2012);14:5.

2. Murlidhar V, Zeinali M, Grabauskiene S, et al. A Radial Flow Microfluidic device for ultra-high-throughput affinity-based isolation of circulating tumor cells. Small. (2014);10:4895.

3. Edwards DP, Grzyb KT, Dressler LG, et al. Monoclonal antibody identification and characterization of a $\mathrm{Mr} 43,000$ membrane glycoprotein associated with human breast cancer. Cancer Res. (1986);46:1306.

4. Piyathilake CJ, Frost AR, Weiss H, et al. The expression of Ep-CAM(17-1A) in squamous cell cancers of the lung. Hum Pathol. (2000);31(4):482-7.

5. Sansonno D, Dammacco F. Expression and distribution of a human colon-carcinoma-associated antigen in normal and diseased liver tissue. Pathobiology. (1993);61:193.

6. de Boer CJ, Van Krieken J, Janssen-Van Rhijn CM, et al. Expression of Ep-CAM in normal, regenerating, metaplastic, and neoplastic liver. J Pathol. (2000);191:102.

7. Ruck P, Wichert G, Handgreteinger R, et al. Symposium: respiration in birds. respiration in birds. J Pathol. (2000); 191(1):102-3.

8. Zhang SL, Zhang HS, Reuter VE, et al. Expression of poytential target antigens for immunotherapy on primary and metastatic prostate cancers. Clin. Cancer Res. (1998); 4:295.

9. Poczatek RB, Myers RB, Manne U, et al. Ep-Cam levels in prostatic adenocarcinoma and prostatic intraepithelial neoplasia. J Urol. (1999);162:1462.

10. Szala S, Froehlich M, Scollon M, et al. Molecular cloning of cDNA for the carcinoma-associated antigen GA733-2. Natl Acad Sci. (1990);87:3542.

11. Herlyn M, Steplewski Z, Herlyn D, et al. Colorectal carcinoma-specific antigen: detection by means of monoclonal antibodies. Proc Natl Accad Sci. USA (1979); 76:1438.
12. Kumble S, Omary MB, Fajardo LF, et al. Multifocal heterogeneity in villin and Ep-CAM expression in Barrett's esophagus. Int J Cancer (1996);66: 48.

13. Martin IG, Cutts SG, Birbeck K, et al. Expression of the 17-1A antigen in gastric and gastro-oesophageal junction adenocarcinomas: A potential immunotherapeutic target? J Clin Pathol. (1999);52:701.

14. Kirby BJ, Jodari M, Loftus MS, et al. Function characterization of circulating tumor cells with a prostatecancer-specific microfluidic device. Plos One (2012); 7:e35976.

15. Adams AA, Okagbare PI, Feng J, et al. Highly efficient circulating tumor cell isolation from whole blood and label-free enumeration using polymer-based microfluidics with an integrated conductivity sensor. J Am Chem Soc. (2008);130:8633.

16. Mikolajczyk SD, Millar LS, Tsinberg P, et al. Detection of EpCAM-negative and Cytokeratin-negative circulating tumor cells in peripheral blood. J Oncol. (2011);252361.

17. Nagrath S, Sequist LV, Maheswaran S, et al. Isolation of rare circulating tumour cells in cancer patients by microchip technology. Nature (2007);450:1235.

18. Stott SL, Hsu CH, Tsukrov DI, et al. Isolation of circulating tumor cells using a microvortex-generating herringbone-chip. Proc. Natl Acad Sci. (2010);107:18392.

19. Yoon HJ, Kim TH, Zhang Z, et al. Sensitive capture of circulating tumor cells by functionalized graphene oxide nanosheets. Nat Nanotechnol. (2013);8:735.

20. Zhao L, Tang $\mathrm{C}, \mathrm{Xu} \mathrm{L}$, et al. Enhanced and differential capture of circulating tumor cells from lung cancer patients by microfluidic assays using aptamer cocktail. Small (2016);12:1072.

21. Morton KJ, Sturm JC, Austin RH, et al. Nanoimprinted fluidic device for continuous separation of nanoparticles. Proc of $\mu$ TAS. (2006); 1014 .

22. Davis JA, Inglis DW, Morton KJ, et al. Deterministic hydrodynamics: Taking blood apart. Natl Acad Sci. (2006);103:14779.

23. Loutherback K, Silva JD, Liu L, et al. Deterministic separation of cancer cells from blood at $10 \mathrm{~mL} / \mathrm{min}$. AIP Advances. (2012);2:042107. 
24. Alshareef M, Metrakos N, Perez EJ, et al. Separation of tumor cells with dielectrophoresis-based microfluidic chip. Biomicrofluidics. (2013); 7: 011803.

25. Anas A, Ion S, Rama B, et al. Interdigitated comb-like electrodes for continuous separation of malignant cells from blood using dielectrophoresis. Electrophoresis. (2011);32:1327.

26. $\mathrm{Yu} \mathrm{M}$, Stott S, Toner M, et al. Circulating tumor cells: approaches to isolation and characterization. J Cell Biol (2011);192:373.

27. Du Z, Cheng K, Vaughn M, et al. Recognition and capture of breast cancer cells. Biomed micro devices (2007);9:35.

28. Shao N, Wickstrom E, Panchapakesan B. Nanotubeantibody biosensor arrays for the detection of circulating breast cancer cells. Nanotechnology (2008);19:465101.

29. Chung J, Issadore D, Ullal A, et al. Rare cell isolation and profiling on a hybrid magnetic/size-sorting chip. Biomicrofluidics (2013); 7:9.

30. Yang L, Lang JC, Balasubramanian P, et al. Optimization of an enrichment process for circulating tumor cells from the blood of head and neck csancer patients through depletion of normal cells. Biotechnol. Bioeng. (2009); 102:521.

31. Liu Z, Fusi A, Klopocki E, et al. Enrichment of circulating tumor cells by immunomagnetic nanobeads from peripheral blood of cancer patients: a method of unbiased enumeration and characterization. J Transl Med. (2011); 9:70.

32. Meye A, Bilkenroth U, Schmidt U, et al. Isolation and enrichment of urologic tumor cells in blood samples by a semi-automated CD45 depletion autoMACS protocol. Int $\mathrm{J}$ Oncol. (2002);21:521.

33. Zigeuner RE, Riesenberg $\mathrm{R}$, Pohla $\mathrm{H}$, et al. Isolation of circulating cancer cells from whole blood by immunomagnetic cell enrichment and unenriched immunocytochemistry in vitro. J Urol (2003);169:701.

34. Farokhzad OC, Jon SY, Khademhosseini A, et al. Nanoparticle-aptamer bioconjugates: a new approach for targeting prostate cancer cell. Cancer Res. (2004);64:7668.

35. Song KM, Lee S, Ban C. Aptamers and their applications. Sensors. (2012);12:612.

36. Stott SL, Hsu CH, Tsukrov DI, et al. Isolation of circulating tumor cells using a microvortex-generating herringbone-chip. Proc Natl Acad Sci. USA (2010);18392.

37. Wang S, Liu K, Liu J, et al. Highly efficient capture of circulating tumor cells by using nanostructured silicon substrates with integrated chaotic micromixers. Angew Chem Int Ed. (2011);50:3084.

38. Sheng W, Ogunwobi OO, Chen T, et al. Capture, release and culture of circulating tumor cells from pancreatic cancer patients using an enhanced mixing chip. Lab chip. (2014);14:89.

39. Yoon HJ, Kim TH, Zhang Z, et al. Sensitve capture of circulating tumour cells by functionalized graphene oxide nanosheets. Nat Nanotechnol. (2013);8:185.

40. Jeon S, Hong W, Lee ES, et al. High-purity isolation and recovery of circulating tumor cells using conducting polymer-deposited microfluidic device. Theranostics. (2014);4:1123.

41. Zhao L, Tang $\mathrm{C}, \mathrm{Xu} \mathrm{L}$, et al. Enhanced and differential capture of circulating tumor cells from lung cancer patients by microfluidic assays using aptamer cocktail. Small (2016);12:1072.

42. Zheng F, Cheng Y, Wang J, et al. Aptamer-functionalized barcode particles for the capture and detection of multiple types of Circulating Tumor Cells. Adv Mater. (2014); 26:7333.

43. Jeon S, Hong W, Lee ES, et al. High-purity isolation and recovery of circulating tumor cells using conducting polymer-deposited microfluidic device. Theranostics. (2014); 4:1123.

44. Deng Y, Zhang Y, Sun S, et al. An integrated microfluidic chip system for single-cell secretion profiling of rare circulating tumor cells. Scientific Reports. (2014);4:7499.

45. Adams AA, Okagbare P, Feng J, et al. Highly efficient circulating tumor cell isolation from whole blood and lsabel-free enumeration using polymer-based microfluidics with an integrated conductivity sensor. J Am Chem Soc. (2008);130:8633.

46. Saucedo-Zeni N, Mewes S, Niestroj R, et al. A novel method for the in vivo isolation of tumor cells from peripheral blood of cancer patients using a functionalized and structured medical wire. Int J Oncol. (2012);41:1241.

47. Gleghorn JP, Pratt ED, Denning D, et al. Capture of circulating tumor cells from whole blood of prostate cancer patients using geometrically enhanced differential immunocapture (GEDI) and prostate-specific antibody. Lab chip. (2010);10:27.

48. Shen Q, Xu L, Zhao L, et al. Specific capture and release of circulating tumor cells using aptamer modified nanosubstrates. Adv Mater. (2013);25: 2368.

49. $\mathrm{Xu} \mathrm{Y,} \mathrm{Phillips} \mathrm{JA,} \mathrm{Yan} \mathrm{J,} \mathrm{et} \mathrm{al.} \mathrm{Aptamer-based}$ microfluidic device for enrichment, sorting, and detection of multiple cancer cells. Small. (2016);12:1072.

50. Kumeria T, Kurkuri MD, Diener KR, et al. Label-free reflecometric interference microchip biosensor based on nanoporous alumina for detection of circulating tumour cells. Losic, Biosens Bioelectron. (2012);35:167.

51. Philips JA, Xu Y, Xia Z, et al. Enrichment of cancer cells using aptamers immobilized on a microfluidic channel. Anal Chem. (2009);81:1033.

52. Deng Y, Zhang Y, Sun S, et al. An integrated microfluidic chip system for single-cell secretion profiling of rare circulating tumor cells. Sci Rep. (2014);4:7499.

\section{*Correspondence to}

Hongmei Chen

Division of Nanobionic Research, Suzhou Institute of NanoTech and Nano-Bionics, Chinese Academy of Sciences, Suzhou, Jiangsu, 215123, China

Institute of Semiconductors, Chinese Academy of Sciences, Beijing, 100083, China 
Citation: Chen H, Cao B, Li L. Immunoaffinity binding for separation of circulating tumor cells with microfluidic chips. J Mol Oncol Res. 2017;1(1):5-15.

E-mail: hongmeichen@semi.ac.cn 